\title{
Force Magnitude Reconstruction Using the Force Transmissibility Concept
}

\author{
Y. E. Lage, N. M. M. Maia, and M. M. Neves \\ LAETA, IDMEC, Instituto Superior Técnico, Universidade de Lisboa, Avenida Rovisco Pais, 1049-001 Lisboa, Portugal \\ Correspondence should be addressed to N. M. M. Maia; nmaia@dem.ist.utl.pt
}

Received 6 November 2013; Revised 14 March 2014; Accepted 16 March 2014; Published 15 April 2014

Academic Editor: Reza Jazar

Copyright (C) 2014 Y. E. Lage et al. This is an open access article distributed under the Creative Commons Attribution License, which permits unrestricted use, distribution, and reproduction in any medium, provided the original work is properly cited.

\begin{abstract}
This paper proposes the reconstruction of forces, based on the direct and inverse problems of transmissibility in multiple degree of freedom (MDOF) systems. The objective and novelty are to use the force transmissibility to calculate reactions given the applied loads (and vice versa). This method, relating two sets of forces, proves to be an alternative to the common inverse problem based on the measurement of FRFs and operational accelerations to determine operational forces, as it can be advantageous in some cases. This implies the a priori knowledge of the transmissibility of the structure, either experimentally or numerically. In this study a finite element model is built, describing with enough accuracy the dynamic behavior of the structure. The numerical model will play a key role in the construction of the transmissibility matrix; this will be used to evaluate either the reaction or the applied forces, using experimental data. This constitutes a hybrid methodology, which is validated experimentally. The authors present several comparisons between reconstructed and experimentally measured sets of forces. It is shown that the proposed method is able to produce good results in the reconstruction of the forces, underlining its potential for other structures and possible applications.
\end{abstract}

\section{Introduction}

The estimation of excitation forces is an important issue in the dynamic analysis of structures; whenever possible, force transducers are used for the direct measurement. However, such a direct measurement is not possible or feasible in many situations; take for example the case of forces transmitted by machinery, like a compressor transmitting to the foundation. Moreover, the determination of the loads of a mechanic system is of unquestionable interest as an efficiency and diagnostics tool. Whenever the direct knowledge of the force is not possible, one proceeds by the inverse way. From the measurement of a set of dynamic responses along the structure (e.g., acceleration, displacement, or strain) it can be possible to estimate the forces indirectly. Therefore, one follows an inverse process and, under such circumstances, force identification is an inverse problem. In this inverse problem, only a finite number of points of the structure can be measured and such a limitation makes the problem ill-posed $[1,2]$.
Force identification can be performed either in the time domain or (more often) in the frequency domain. Therefore, the force identification problem includes (i) the choice of the domain to use, (ii) the localization of the forces, and (iii) their reconstruction.

Stevens [3] and more recently Hundhausen [4] tried to clarify the inverse problem and presented a review of practical methods capable of solving in a satisfactory way the inverse problem for the case of linear vibration systems. Other works in the last few decades have been made in the sense of achieving a practical method capable of solving this problem [5-11].

In [12] some reconstruction techniques are used, like the inverse structural filter (ISF), the delayed multistep ISF, the weighted accelerations technique (SWAT), and the frequency domain inverse method (FD), where it is necessary to know a priori the force locations.

In [13] a method is proposed to solve the inverse problem in two sequential steps: in the first step, a transmissibility model for multiple degree of freedom systems is used to assess 
the location and number of independent loads; once that is accomplished, the frequency response function (FRF) matrix of the structure is used to regenerate the load vector. In this paper the authors propose another form to estimate forces, consisting of using the force transmissibility properties to estimate specific sets of forces. For that, it is necessary to understand the transmissibility concept, which can be divided into motion transmissibility or force transmissibility. One of the first approaches for the generalization of the transmissibility to MDOF systems was presented in 1998 [14]. In [15] an extension of the acceleration transmissibility for MDOF is presented and used to predict accelerations. Those works were followed by [16, 17], which present a more consistent generalization of the concept for structures with multiple degrees of freedom. In [18], the authors compared the transmissibility matrix obtained by spectral densities of the responses with those coming from the FRFs, proving to be the same and independent of the applied forces. In [19] the authors present the experimental evaluation of the transmissibility concept for MDOF systems. In addition to the developments in the formulation of this problem one can find a wide range of problems in which one can apply this concept [20,21]. In [22] transmissibility is used in a structural optimization problem for reducing vibration and noise and in [23] it is used as indicator of structural damage.

This paper is focused on the development of the concept of transmissibility between two sets of forces (reactions and applied forces). In the single degree of freedom system the force transmissibility is defined as the ratio between the magnitude of the transmitted force (ground reaction) and the applied force. This concept has been generalized for MDOF systems considering relations between two sets of dynamic loads [24]. The authors explain the formulation based on the dynamic stiffness matrices and also on the receptance matrices. In [25] the authors present a method to predict the unknown dynamical forces, analyzing a function error that compares the known reaction forces and the values calculated using the properties of transmissibility. It is also possible to find a recent review of the two types of transmissibility discussed here in [26].

The present work aims to demonstrate experimentally the force transmissibility concept and its use in force reconstruction, using numerical transmissibility models and experimental measured forces. This method does not replace the common inverse problem based on the measurement of FRFs and operational accelerations to determine operational forces, but it can be an alternative method with particular advantages when one can only measure a set of forces, without the need of accelerometers. Results for the direct calculations of reactions by force transmissibility are presented, as well as for the inverse problem. For the experiments, a beam supported in both extremities and subjected to a set of concentrated loads is used. A comparison between the theoretical transmissibility and the experimentally measured one is also presented. The results show a comparison between the reconstructed set of forces (reactions or applied forces) using a numerical transmissibility with the correspondent experimentally measured set. One of the objectives of this work is to demonstrate the efficiency of the method extended

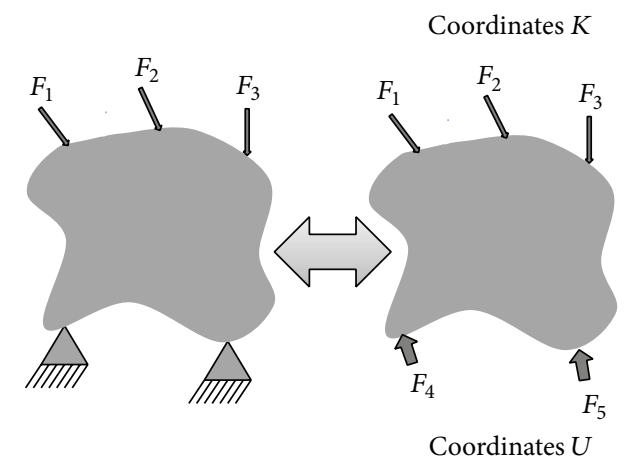

FIGURE 1: Original supported structure and equivalent structure in dynamic equilibrium.

to MDOF systems and how simple it is to apply the procedure in the context of a machine monitoring process.

\section{Transmissibility of Forces in MDOF Systems}

For a single degree of freedom, the transmissibility is defined as the ratio between the magnitude of the transmitted load (the ground reaction) and the magnitude of the applied load, for harmonic excitation.

For a MDOF linear elastodynamic system, the concept consists of establishing a relation between the set of the reactions and the set of harmonically applied forces, where the displacement constraints are replaced by the corresponding set of reactions forces, as illustrated in Figure 1.

One defines two groups of forces. One of them contains the reactions $\mathbf{F}_{U}$, which are obtained replacing the displacement constraints of the structure, assuming those displacements as equal to zero. The second group includes the forces applied to the structure, $\mathbf{F}_{K}$. In [24] those two groups of forces are denoted by "unknown set of loads" and "known set of loads," respectively, while in our case, we designate them only by forces $(K)$ and reactions $(U)$, as the objective is the resolution of the direct and inverse problems. In the direct problem, for the evaluation of the reactions, the applied loads constitute the known set. In the inverse problem, for the estimation of the applied loads, the known set is constituted by the reactions. Once these two groups of forces are defined, the transmissibility is established by relating both groups.

2.1. Transmissibility of Forces in Terms of FRFs. The dynamic amplitude response of a MDOF system to a harmonic excitation can be described by

$$
\mathbf{Y}(\omega)=\mathbf{H}(\omega) \mathbf{F}(\omega),
$$

where $\mathbf{Y}(\omega)$ is the vector of the dynamic displacement amplitudes, $\mathbf{H}(\omega)$ is the receptance matrix, $\mathbf{F}(\omega)$ is the vector of the external force amplitudes, and $\omega$ is the excitation frequency. Equation (1) can be rewritten grouping the degrees of freedom corresponding to the applied forces with the index $K\left(n_{K}\right.$ DOFs $)$ and the ones corresponding to the reaction forces with the index $U\left(n_{U}\right.$ DOFs). If the degrees of freedom where no forces exist are rejected, one may reduce (1) to 
the following relation between dynamical displacements and their excitation forces:

$$
\left\{\begin{array}{c}
\mathbf{Y}_{K} \\
\mathbf{Y}_{U}
\end{array}\right\}=\left[\begin{array}{ll}
\mathbf{H}_{K K} & \mathbf{H}_{K U} \\
\mathbf{H}_{U K} & \mathbf{H}_{U U}
\end{array}\right]\left\{\begin{array}{l}
\mathbf{F}_{K} \\
\mathbf{F}_{U}
\end{array}\right\} .
$$

As $\mathbf{F}_{U}$ represents the reaction forces, the displacements at the corresponding coordinates may be made equal to zero; that is, $\mathbf{Y}_{U}=\mathbf{0}$, and for this condition it follows that

$$
\mathbf{0}=\mathbf{H}_{U K} \mathbf{F}_{K}+\mathbf{H}_{U U} \mathbf{F}_{U}
$$

From (3), one can obtain the relation between reaction and applied forces:

$$
\mathbf{F}_{U}=-\left(\mathbf{H}_{U U}\right)^{-1} \mathbf{H}_{U K} \mathbf{F}_{K}
$$

The force transmissibility matrix can thus be defined as

$$
\left(\mathbf{T}_{f}\right)_{U K}=-\left(\mathbf{H}_{U U}\right)^{-1} \mathbf{H}_{U K}
$$

The force transmissibility defined in (5) relates the reactions $\mathbf{F}_{U}$ where the displacements are zero to the forces $\mathbf{F}_{K}$ applied to the structure.

If the displacements at the coordinates of the reactions are not zero, that is, $\mathbf{Y}_{U} \neq \mathbf{0}$, one can write the following relation:

$$
\mathbf{F}_{U}=\mathbf{H}_{U U}^{-1}\left(\mathbf{Y}_{U}-\mathbf{H}_{U K} \mathbf{F}_{K}\right)
$$

and using (5), one obtains

$$
\mathbf{F}_{U}=\left(\mathbf{T}_{f}\right)_{U K} \mathbf{F}_{K}+\left(\mathbf{H}_{U U}\right)^{-1} \mathbf{Y}_{U}
$$

2.2. Transmissibility of Forces in Terms of Dynamic Stiffness. Using the dynamic stiffness matrix, the MDOF system can be described through the following equation:

$$
\mathbf{Z}(\omega) \mathbf{Y}(\omega)=\mathbf{F}(\omega)
$$

Following the same formulation made for the receptance in the previous subsection, but adding a group of fictitious forces $\mathbf{F}_{C}$ at the $n_{C}$ remaining coordinates, (8) can be expanded, becoming

$$
\left[\begin{array}{lll}
\mathbf{Z}_{K K} & \mathbf{Z}_{K U} & \mathbf{Z}_{K C} \\
\mathbf{Z}_{U K} & \mathbf{Z}_{U U} & \mathbf{Z}_{U C} \\
\mathbf{Z}_{C K} & \mathbf{Z}_{C U} & \mathbf{Z}_{C C}
\end{array}\right]\left\{\begin{array}{l}
\mathbf{Y}_{K} \\
\mathbf{Y}_{U} \\
\mathbf{Y}_{C}
\end{array}\right\}=\left\{\begin{array}{l}
\mathbf{F}_{K} \\
\mathbf{F}_{U} \\
\mathbf{F}_{C}
\end{array}\right\} .
$$

Then, rearranging the system in order to group the set $K$ together with the remaining $C$ DOFs in a new group with subindex $E$, one gets the following:

$$
\left[\begin{array}{ll}
\mathbf{Z}_{E E} & \mathbf{Z}_{E U} \\
\mathbf{Z}_{U E} & \mathbf{Z}_{U U}
\end{array}\right]\left\{\begin{array}{c}
\mathbf{Y}_{E} \\
\mathbf{Y}_{U}
\end{array}\right\}=\left\{\begin{array}{c}
\mathbf{F}_{E} \\
\mathbf{F}_{U}
\end{array}\right\} .
$$

With this in mind and assuming once again zero displacements at the supports $\left(\mathbf{Y}_{U}=\mathbf{0}\right)$, one can write the following equalities:

$$
\begin{aligned}
& \mathbf{Z}_{E E} \mathbf{Y}_{E}=\mathbf{F}_{E} \\
& \mathbf{Z}_{U E} \mathbf{Y}_{E}=\mathbf{F}_{U} .
\end{aligned}
$$

From (11), one can obtain the relation between the two force vectors, reactions, and applied forces (plus fictitious forces):

$$
\mathbf{F}_{U}=\mathbf{Z}_{U E}\left(\mathbf{Z}_{E E}\right)^{-1} \mathbf{F}_{E} \quad \text { with } \mathbf{F}_{E}=\left\{\begin{array}{c}
\mathbf{F}_{K} \\
\mathbf{0}
\end{array}\right\} \text {. }
$$

This force transmissibility relates the loads applied to the structure $\mathbf{F}_{K}$ with the corresponding reactions $\mathbf{F}_{U}$. From (12) an expression for the force transmissibility matrix is obtained:

$$
\left(\mathbf{T}_{f}\right)_{U E}=\mathbf{Z}_{U E}\left(\mathbf{Z}_{E E}\right)^{-1}
$$

Note that only the columns of $\left(\mathbf{T}_{f}\right)_{U E}$ corresponding to $\mathbf{F}_{K}$ are relevant to the transmissibility between the two sets of forces. The remaining columns multiply by zero, and so one obtains the submatrix $\left(\mathbf{T}_{f}\right)_{U K}$ presented in the previous section.

This is a simple expression and it certainly can be useful in a number of cases, as long as a complete discretization of the structure is available. In the present work, the force transmissibility matrix is used in the reconstruction of the reactions and of the applied forces, using a finite element model.

\section{Hybrid Methodology for Force Reconstruction}

The main objective of this paper is the reconstruction of the reaction or applied forces in the structure using the transmissibility concept.

Here one considers two types of problems involving the estimation of forces:

(1) reaction forces estimation (direct problem) with the objective to calculate a set of unknown reactions using information from the known set of applied loads:

$$
\mathbf{F}_{U}=\left(\mathbf{T}_{f}\right)_{U K} \mathbf{F}_{K}
$$

(2) applied forces estimation (inverse problem) with the objective of calculating a set of applied forces using information from the known set of reaction forces:

$$
\mathbf{F}_{K}=\left(\left(\mathbf{T}_{f}\right)_{U K}\right)^{+} \mathbf{F}_{U},
$$

where the superscript + stands for pseudoinverse. The method to estimate the applied forces is limited by the number of reactions. So, it is not possible to perform the pseudoinverse of the transmissibility matrix if the number of applied forces is greater than the number of reactions. In this case, the condition to perform the pseudoinverse is $\# U \geq \# K$.

In both situations (14) and (15), the calculation involves a vector of forces and a transmissibility matrix or its pseudoinverse and this requires the knowledge of the transmissibility matrix. Therefore, one recurs to the numerical modeling to obtain the required transmissibility matrix.

One calls this methodology a hybrid one, because it combines a numerical model to describe the structure (to 
build the transmissibility matrix) and a set of experimentally measured forces.

The transmissibility matrix could also be obtained from experimental data using the ratio between the simulated reactions and the applied force one by one. Alternatively, this could be estimated from experimental data by modal identification of the structure and for that it would be necessary to measure the FRFs required to build the specific receptance matrices in a freely suspended system (usually more difficult to perform).

One assumes here to be possible to create a numerical model to produce the receptance matrix $\mathbf{H}$ capable of reproducing with enough accuracy its dynamic behavior. This numerical model could be created using the finite element method and updated with experimental data (for example, experimentally measured FRF, not necessarily related to the sets of applied or reactions forces). Once the numerical model has been built, this is used for the calculation of the required transmissibility matrix and then together with a set of experimental data, it is possible to estimate the desired vector $\mathbf{F}_{U}\left(\right.$ or $\left.\mathbf{F}_{K}\right)$.

3.1. Finite Element Model. In this paper, we consider only harmonic discrete loads and assume that the model of the structure is known. In this context, the dynamic stiffness matrix $\mathbf{Z}(\omega)$ may be built through FEM. The dynamic stiffness matrix is defined as follows:

$$
\mathbf{Z}(\omega)=\mathbf{K}-\omega^{2} \mathbf{M}+\mathbf{i} \omega \mathbf{C},
$$

where $\mathbf{K}, \mathbf{M}$, and $\mathbf{C}$ represent the stiffness, mass, and proportional damping matrix, with $\mathbf{C}=\alpha \mathbf{K}+\beta \mathbf{M} ; \alpha$ and $\beta$ are constants to be evaluated experimentally. Often, one works with the receptance matrix $\mathbf{H}$, which is the inverse of dynamic stiffness matrix $\mathbf{Z}$ :

$$
\mathbf{H}(\omega)=\mathbf{Z}^{-1}(\omega)
$$

Taking into account the orthogonality conditions using the mass-normalized modes $\phi$, one has

$$
\begin{aligned}
\phi^{\mathrm{T}} \mathbf{M} \phi & =\mathbf{I}, \\
\phi^{\mathrm{T}} \mathbf{K} \phi & =\operatorname{diag}\left(\omega_{r}^{2}\right), \\
\phi^{\mathrm{T}} \mathbf{C} \phi & =\operatorname{diag}\left(2 \xi_{r} \omega_{r}\right),
\end{aligned}
$$

where $\omega_{r}$ are the natural frequencies and $\xi_{r}$ are the damping factors. Substituting (18) in (16) and considering (17), (1) becomes

$$
\mathbf{Y}(\omega)=\phi\left(\operatorname{diag}\left(\omega_{r}^{2}-\omega+i 2 \xi_{r} \omega \omega_{r}\right)\right)^{-1} \phi^{\mathrm{T}} \mathbf{F}(\omega) .
$$

A computer program was developed (in Matlab environment) to build the stiffness, mass, and damping matrices, using the finite element method, for the given discretized structure into $N$ Bernoulli-Euler 2D beam elements with 2 nodes each, resulting in $N N=N+1$ nodes in freely suspended conditions. Each node has 3 degrees of freedom $(x, y, \theta)$ and so the matrix order is $3 \times N N$.

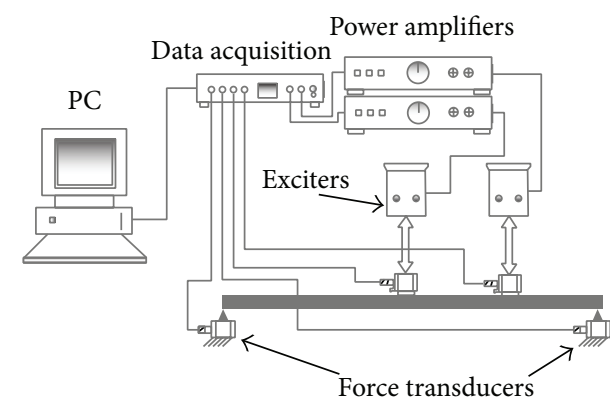

FIGURE 2: Illustration of the experimental setup developed for the study of force transmissibility.

TABLE 1: Beam properties.

\begin{tabular}{lc}
\hline Young's modulus- $E$ & $208 \mathrm{GPa}$ \\
Density $-\rho$ & $7840 \mathrm{~kg} / \mathrm{m}^{3}$ \\
Length $-L$ & $0.8 \mathrm{~m}$ \\
Section width $-b$ & $5.0 \times 10^{-3} \mathrm{~m}$ \\
Section height $-h$ & $20.0 \times 10^{-3} \mathrm{~m}$ \\
Second moment of area- $-I$ & $2.0883 \times 10^{-10} \mathrm{~m}^{4}$ \\
\hline
\end{tabular}

3.2. Experimental Setup. The experimental setup is presented in Figure 2.

For the experimental tests, the following equipment was used:

(i) vibration exciter Bruell \& Kjaer Type 4809;

(ii) power amplifier Bruell \& Kjaer Type 2706;

(iii) force transducer PCB PIEZOTRONICS Model 208C01;

(iv) data acquisition equipment Bruell \& Kjaer Type 3560C;

(v) piezoelectric accelerometer Bruell \& Kjaer Type 4507.

\section{Experimental Application}

In this section, one presents a discussion on the experimental tests performed with different types of supports and results for force reconstruction. Once the type of support to be used in the experimental tests for a simply supported beam has been chosen, one compares results for the experimental and numerical transmissibility matrix in the case of a single applied force. The transmissibility model for simply supported conditions is validated and the direct and inverse problems are solved in the case with one or two applied forces and two reactions. In all cases the excitation signal is a multisine signal transmitted to the exciter, which reproduces an excitation with constant amplitude in frequency.

4.1. FE Beam Model. For the performed tests, a steel beam with uniform rectangular crosssection is used, subjected to a set of forces. The properties of the beam are presented in Table 1 . The structure is discretized in 16 FEs with equidistant 


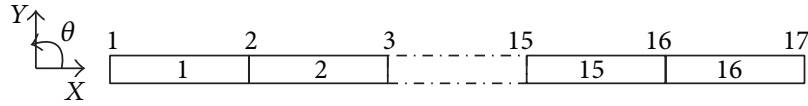

FIgURE 3: Discretized steel beam.

nodes (Figure 3), using 2D Bernoulli-Euler beam elements with 2 nodes and 3 d.o.f. each $(x, y, \theta)$.

The FE model is updated including a proportional damping model to find the best fit to the experimental FRF data. The FE model of the beam is built for freely supported conditions and will be used for the construction of the transmissibility matrix; the update procedure of this model is part of another work presented in [13], so here one starts from the updated model.

4.2. Testing Supports and Receptance Comparison. Here, two different types of supports are compared by FRF measuring, with the objective of confirming that the supports recreate the conditions of a simply supported beam. These tests are somewhat outside the scope of this work, but they are nonetheless important for the validation of the numerical model. To do this, a simple supported steel beam is subjected to one applied force $\mathbf{F}_{K}$ at node 7 and two reactions $\mathbf{F}_{U}$ at the nodes 1 and 17, as schematically illustrated in Figure 4. One only considers forces in the $y$ direction. The beam structure is modeled by $2 \mathrm{D}$ Bernoulli-Euler beam FEs, as explained in the previous subsection.

In Figures 5(a) and 5(b) one presents the two types of supports, with the purpose of testing these two possible approximations of the numerical model to represent a simple supported beam. With the conditions for a simply supported beam, only the " $y$ " coordinate of each node is considered; one has two types of supports: (i) one of nylon with wide flange shape section and (ii) the other one made of steel with a pin to allow free rotation.

An experimental FRF plot of both types of supports is shown in Figure 6, together with the corresponding FE numerical FRF. The FRF corresponds to an applied force at node 7 and the respective response at node 11. The results for both supports are very similar, and the differences between the numerical model (after updating) and the experimental measurements are considered as being small; that is, although the numerical model is not exact, it represents with enough accuracy the experimental response of the dynamic system. The updating procedure of the model is considered out of the scope of this paper.

From Figure 6, it is clear that both supports represent with good accuracy the simply supported conditions. The supports in nylon have been chosen, as they are easier to mount and test.

4.3. Experimental Application 1-One Applied Force. The first experimental reconstruction case uses the same configuration showed in Figure 4, now with the nylon supports at the beam extremities. It has only one applied force at node 7 and

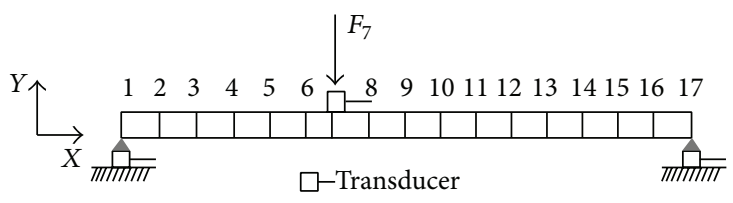

FIGURE 4: Simply supported steel beam showing the positions of the transducers used to measure the applied and reactions forces.

two reactions at nodes 1 and 17 (simple supported beam) as illustrated in Figure 7.

4.3.1. Force Transmissibility. To confirm the proposed concept of force transmissibility presented in Section 2, Figures 8 and 9 show the numerical and experimental transmissibility values for the configuration illustrated in Figure 7. In the case of one applied force and two reactions, the transmissibility matrix has dimensions $2 \times 1$ and can be obtained numerically either using the receptances as proposed in Section 2.1 or the dynamic stiffness, as proposed in Section 2.2. In Figure 8 one can see the transmissibility curve between $F_{7}$ (applied) and $F_{1}$ (reaction) and in Figure 9 the transmissibility between $F_{7}$ (applied) and $F_{17}$ (reaction).

As one can observe (transmissibility directly given by the ratio of the forces), the results obtained numerically using the receptances are very close to the experimental results. Note that around $100 \mathrm{~Hz}$ there is a "bump" in the experimental curve, due to the effect of the lateral modes of the supports of the beam. This effect has not been included in the numerical model as it was not considered as important in the context of the illustration of the method.

4.3.2. Direct Problem-Reaction Estimation. To estimate the reaction forces, one uses the numerical model to set the transmissibility matrix, using (5). Knowing the vector of applied forces, which in this case is simply $F_{7}$, then the calculation of the reactions is given by the following relation:

$$
\left\{\begin{array}{c}
F_{1} \\
F_{17}
\end{array}\right\}=\underset{\substack{\text { (numerical } \\
\text { model) }}}{\left[\begin{array}{c}
T_{1,7} \\
T_{17,7}
\end{array}\right]}\left\{F_{7}\right\} .
$$

From Figures 10 and 11, it is clear that the reconstructed reactions match reasonably well the experimentally measured ones.

4.3.3. Inverse Problem-Applied Force Estimation. For the reconstruction of the applied forces, that is, the inverse problem, one needs to know the vector of reactions $\mathbf{F}_{U}$ and the inverse transmissibility matrix. This calculation takes the following form:

$$
\left\{F_{7}\right\}=\underset{\substack{\text { (numerical } \\
\text { model) }}}{\left[\begin{array}{c}
T_{1,7} \\
T_{17,7}
\end{array}\right]^{+}}\left\{\begin{array}{c}
F_{1} \\
F_{17}
\end{array}\right\} .
$$

Figure 12 shows the reconstruction of the applied force, comparing it with the experimental results. As one can observe both curves match relatively well. 


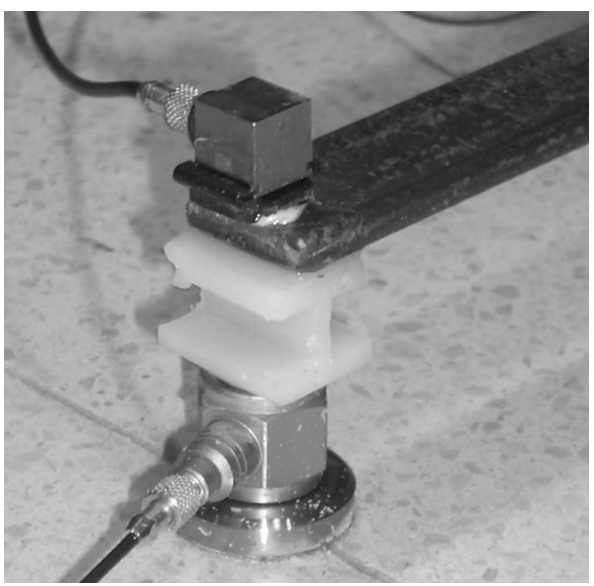

(a) Nylon support

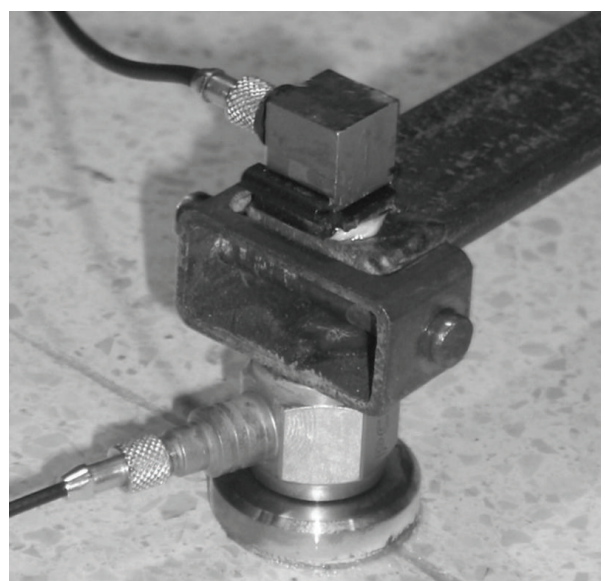

(b) Steel pin support

FigUre 5: Tested supports.

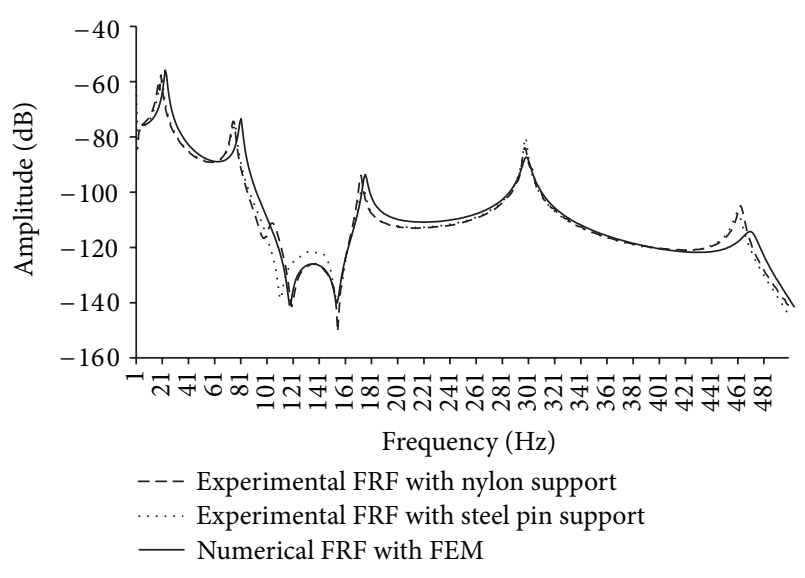

FIGURE 6: Experimental $\operatorname{FRF}_{(11,7) y}$ (for both supports) and numerical $\operatorname{FRF}_{(11,7) y}$.

4.4. Experimental Application 2-Two Applied Forces. For the case with two reactions and two applied forces the transmissibility matrix has dimensions $2 \times 2$. Figure 13 illustrates the experimental setup with two reactions and two applied forces (using the same multisine signal).

Since the procedure for two applied forces is the same as the one presented before for one applied force, the transmissibility matrix is not shown here. As already mentioned, it is also possible to obtain the transmissibility experimentally, applying each force individually and measuring each force ratio. This is due to the fact that the columns of the transmissibility matrix relate to the applied forces independently (5).

4.4.1. Direct Problem-Reaction Estimation. The reconstruction of forces follows the presented methodology:

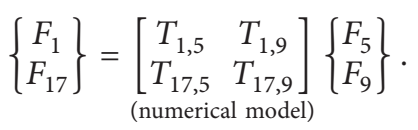



FIGURE 7: Schematic representation of the experimental setup for application 1 .

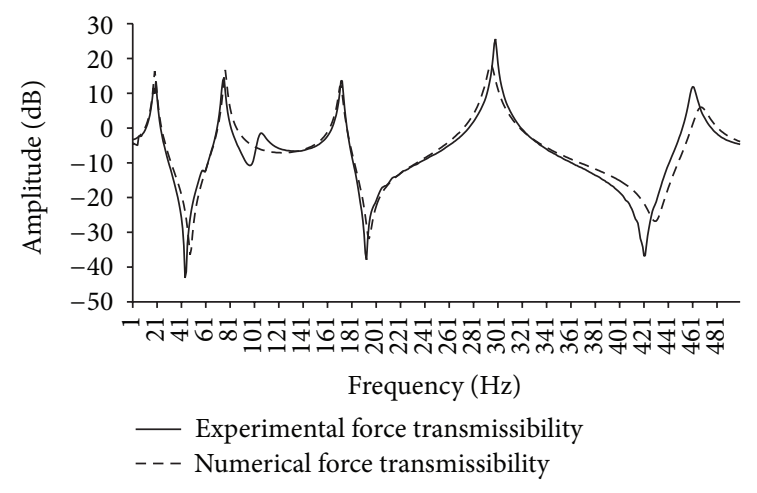

FIGURE 8: Experimental and numerical force transmissibility $T_{1,7}$.

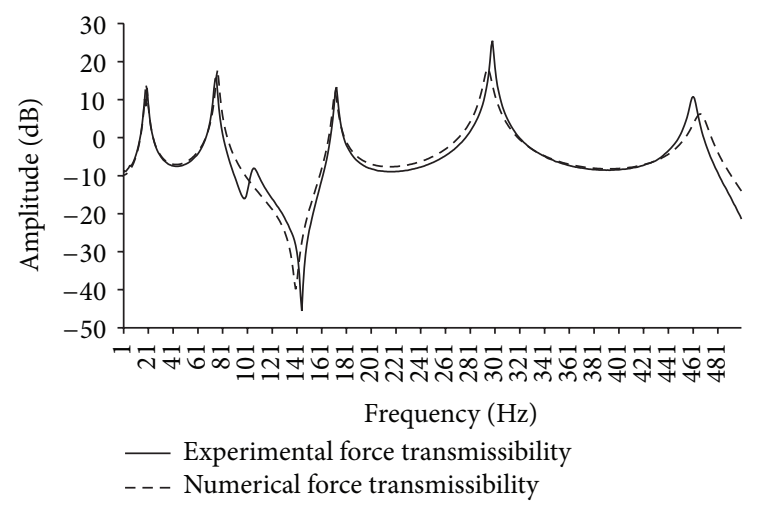

FIgURE 9: Experimental and numerical force transmissibility $T_{17,7}$. 


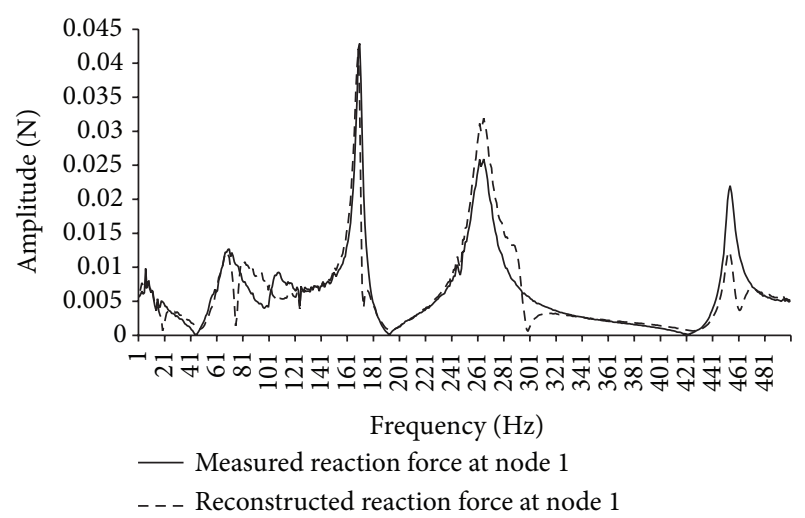

FIGURE 10: Experimental and estimated force reaction $F_{1}$.

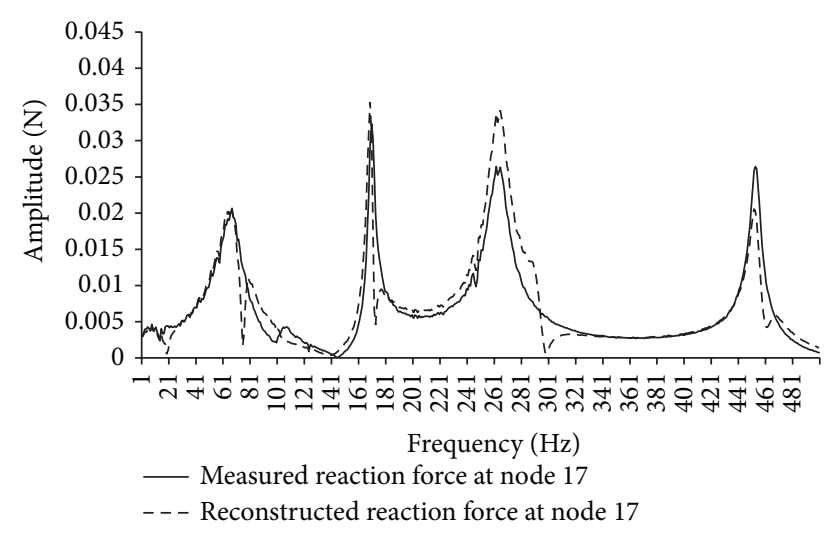

FIGURE 11: Experimental and estimated force reaction $F_{17}$.

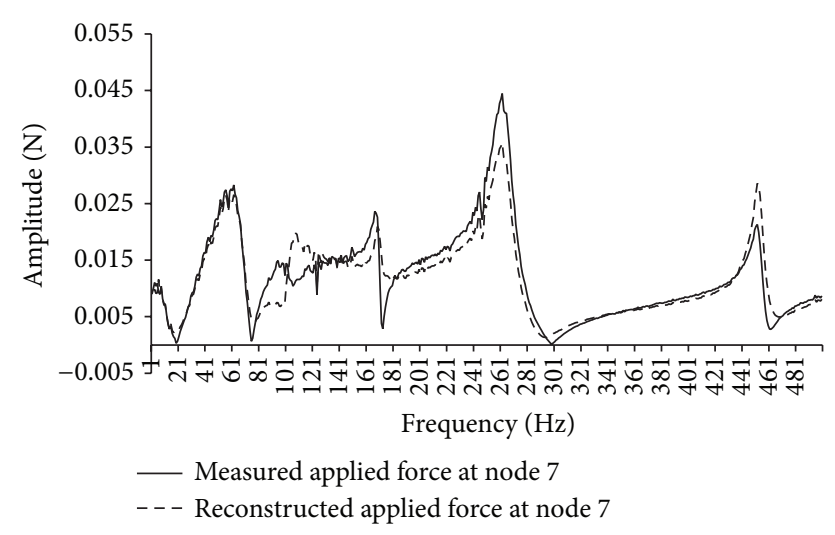

Figure 12: Experimental and estimated applied load $F_{7}$.

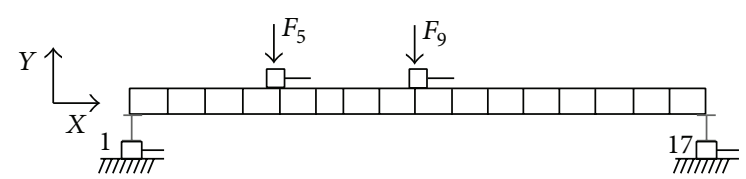

FIGURE 13: Illustration of the experimental setup for application 2.

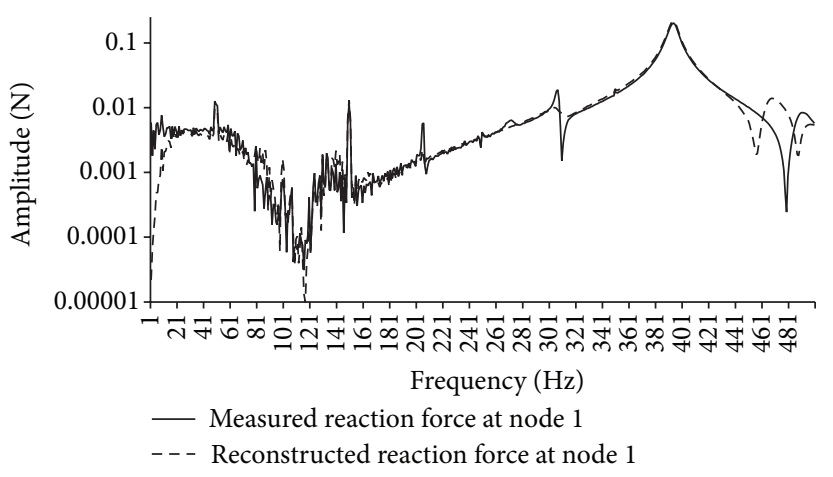

FiguRE 14: Experimental and estimated reaction force $F_{1}$.

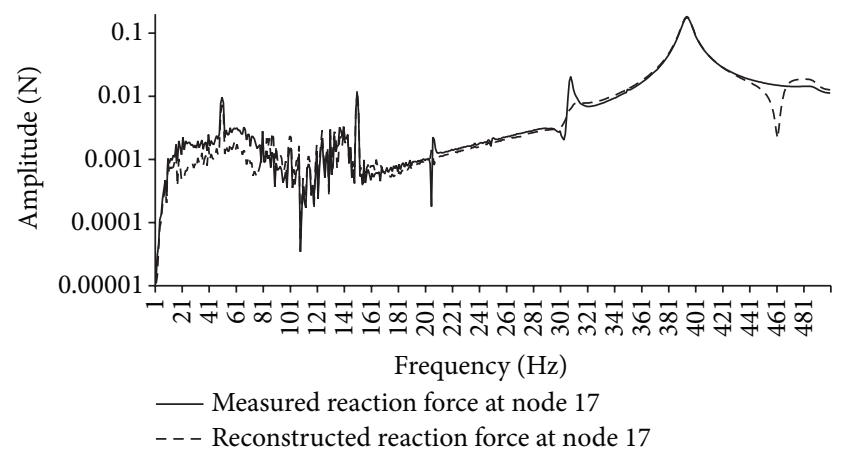

FIGURE 15: Experimental and estimated reaction force $F_{17}$.

The reconstructed values are compared with the experimentally measured ones in Figures 14 and 15.

4.4.2. Inverse Problem-Applied Force Estimation. Now, for the calculation of the applied forces, one has

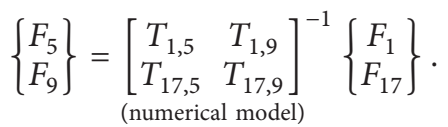

In these last results, for the case with two applied forces and two reactions, one may observe in Figures 16 and 17 several spurious peaks in the force reconstruction $(\approx 50 \mathrm{~Hz}$, $\approx 150 \mathrm{~Hz}, \approx 302 \mathrm{~Hz}$ ). These peaks are due to the small deviations between the numerical model and the real behavior of the structure, namely, the deviations at the antiresonance frequencies. However, in general, a reasonably good approach for the reconstructed forces has been verified in all the studied cases.

With the presented results the force reconstruction using the force transmissibility concept has been validated experimentally, to solve both the direct problem (estimating the reactions) and the inverse problem (estimating the applied forces).

\section{Conclusions}

The force identification method proposed in this paper proved to be an efficient way to estimate reactions or 


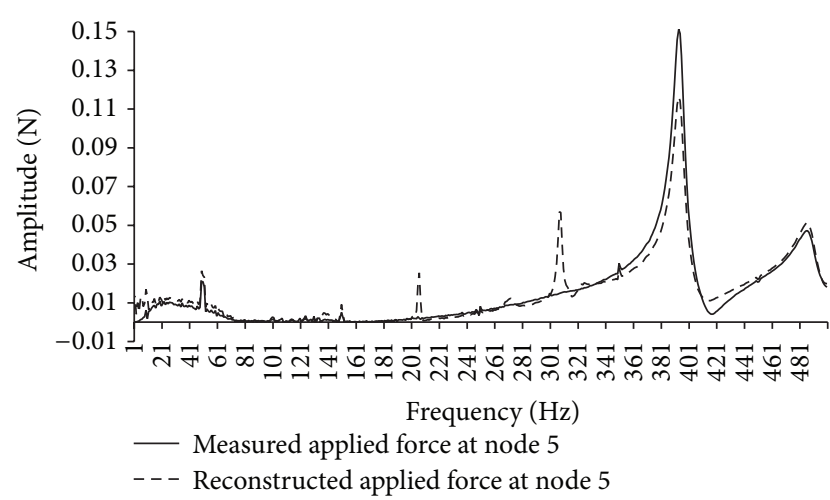

FIgURE 16: Experimental and reconstructed applied load $F_{5}$.

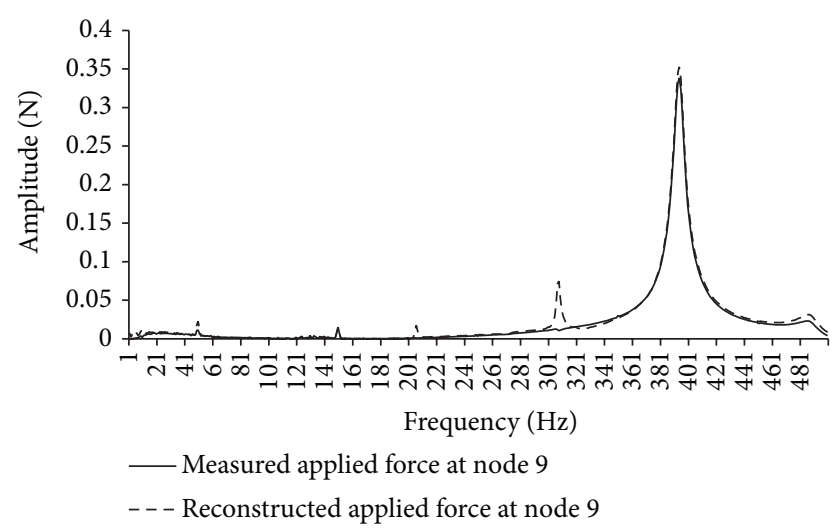

FIGURE 17: Experimental and reconstructed applied load $F_{9}$.

applied forces and an alternative to the use of operational responses. The simplicity of the transmissibility concept is due to the need of only part of the receptance matrix, which can be obtained from a model of the structure in free-free conditions. When using the numerical model it is necessary that the model represents well the true response of the structure; in that way, it is possible to construct any relation of force transmissibility. With this hybrid method and considering a valid model of the structure, one needs only to measure a set of forces to solve the problem. Alternatively, it is possible to estimate previously the experimental matrix of the force transmissibility. All the experimental tests have provided very good results both with the direct and the inverse transmissibility matrices.

The force transmissibility obtained numerically showed to be very close to the experimentally measured one, which is of course of paramount importance for the hybrid force reconstruction method proposed here.

The proposed application of the force transmissibility proved to be able to:

(i) obtain the reaction forces knowing the experimental measured applied forces, which is the direct problem;

(ii) obtain the applied force reconstructed knowing the experimental measured reactions, which is the inverse problem.

\section{Conflict of Interests}

The authors declare that there is no conflict of interests regarding the publication of this paper.

\section{Acknowledgments}

The authors wish to express their gratitude to IDMEC/IST and FCT for the financial support of the research (under Project no. FCT PTDC/EME-PME/71488/2006).

\section{References}

[1] B. Hillary, Indirect measurement of vibration excitation forces [Ph.D. thesis], Imperial College London, Dynamics Section, London, UK, 1983.

[2] B. Hillary and D. J. Ewins, "The use of strain gauges in force determination and frequency response function measurements," in Proceedings of the 2nd International Modal Analysis Conference, pp. 627-634, Orlando, Fla, USA, 1984.

[3] K. K. Stevens, "Force identification problems-an overview," in Proceedings of the 1987 SEM Spring Conference on Experimental Mechanics, pp. 838-844, Houston, Tex, USA, 1987.

[4] R. J. Hundhausen, D. E. Adams, M. Derriso, P. Kukuchek, and R. Alloway, "Transient loads identification for a standoff metallic thermal protection system panel," in Proceedings of the 23rd International Modal Analysis Conference, 2005.

[5] P. Mas, P. Sas, and K. Wyckaert, "Indirect force identification based on impedance matrix inversion: a study on statistical and deterministic accuracy," in Proceedings of 19th International Seminar on Modal Analysis, pp. 1049-1065, 1994.

[6] B. J. Dobson and E. Rider, "A review of the indirect calculation of excitation forces from measured structural response data," Proceedings of the Institution of Mechanical Engineers C: Journal of Mechanical Engineering Science, vol. 204, pp. 69-75, 1990.

[7] C.-K. Ma, J.-M. Chang, and D.-C. Lin, "Input forces estimation of beam structures by an inverse method," Journal of Sound and Vibration, vol. 259, no. 2, pp. 387-407, 2003.

[8] C. Ma and C. Ho, "An inverse method for the estimation of input forces acting on non-linear structural systems," Journal of Sound and Vibration, vol. 275, pp. 953-971, 2004.

[9] A. N. Thite and D. J. Thompson, "The quantification of structure-borne transmission paths by inverse methods, part 1 : improved singular value rejection methods," Journal of Sound and Vibration, vol. 264, no. 2, pp. 411-431, 2003.

[10] A. N. Thite and D. J. Thompson, "The quantification of structure-borne transmission paths by inverse methods, part 2: use of regularization techniques," Journal of Sound and Vibration, vol. 264, no. 2, pp. 433-451, 2003.

[11] H. G. Choi, A. N. Thite, and D. J. Thompson, "A threshold for the use of Tikhonov regularization in inverse force determination," Applied Acoustics, vol. 67, no. 7, pp. 700-719, 2006.

[12] M. S. Allen and T. G. Carne, "Delayed, multi-step inverse structural filter for robust force identification," Mechanical Systems and Signal Processing, vol. 22, no. 5, pp. 1036-1054, 2008.

[13] Y. E. Lage, N. M. M. Maia, M. M. Neves, and A. M. R. Ribeiro, "Force identification using the concept of displacement transmissibility," Journal of Sound and Vibration, vol. 332, no. 7, pp. 1674-1686, 2013.

[14] D. Ewins and W. Liu, "Transmissibility properties of MDOF systems," in Proceedings of the 16th International Modal Analysis 
Conference, pp. 847-854, Santa Barbara, Calif, USA, February 1998.

[15] P. S. Varoto and K. G. McConnell, "Single point vs multi point acceleration transmissibility concepts in vibration testing," in Proceedings of the 16th International Modal Analysis Conference, pp. 83-90, February 1998.

[16] A. M. R. Ribeiro, "On the generalization of the transmissibility concept," in Proceedings of the NATO/ASI Conference on Modal Analysis and Testing, pp. 757-764, 1998.

[17] N. M. M. Maia, J. M. M. Silva, and A. M. R. Ribeiro, “Transmissibility concept in multi-degree-of-freedom systems," Mechanical Systems and Signal Processing, vol. 15, no. 1, pp. 129-137, 2001.

[18] M. Fontul, A. M. R. Ribeiro, J. M. M. Silva, and N. M. M. Maia, "Transmissibility matrix in harmonic and random processes," Shock and Vibration, vol. 11, no. 5, pp. 563-571, 2004.

[19] A. M. R. Ribeiro, N. M. M. Maia, and J. M. M. Silva, "Experimental evaluation of the transmissibility matrix," in Proceedings of the 17th International Modal Analysis Conference, pp. 11261129, February 1999.

[20] A. M. R. Ribeiro, J. M. M. Silva, N. M. M. Maia, and M. Fontul, "Transmissibility in structural coupling," in Proceedings of the International Conference on Noise and Vibration Engineering (ISMA '04), pp. 2707-2716, September 2004.

[21] A. M. R. Ribeiro, M. Fontul, N. M. M. Maia, and J. M. M. Silva, "Further developments on the transmissibility concept for multiple degree of freedom systems," in Proceedings of the 11th International Conference on Vibration Engineering (ISMA '05), pp. 71-76, September 2004.

[22] O. Nobuyuki, T. Takeshi, and A. Kazumassa, "Prediction of transmitted force between components under operating condition for reduction of vibration and noise," in Proceedings of 14th International Seminar on Modal Analysis Conference, 1996.

[23] T. J. Johnson and D. E. Adams, "Transmissibility as a differential indicator of structural damage," Journal of Vibration and Acoustics, vol. 124, no. 4, pp. 634-641, 2002.

[24] N. M. M. Maia, M. Fontul, and A. M. R. Ribeiro, "Transmissibility of forces in multiple-degree-of-freedom systems," in Proceedings of the International Conference on Noise and Vibration Engineering (ISMA '06), 2006.

[25] M. M. Neves and N. M. M. Maia, "Estimation of applied forces using the transmissibility concept," in Proceedings of the International Conference on Noise and Vibration Engineering, pp. 3887-3897, Leuven, Belgium, 2010.

[26] N. M. M. Maia, A. P. V. Urgueira, and R. A. B. Almeida, "Whys and wherefores of transmissibility," in Vibration Analysis and Control-New Trends and Developments, F. Beltran-Carbajal, Ed., chapter 10, pp. 197-216, InTech, 2011. 

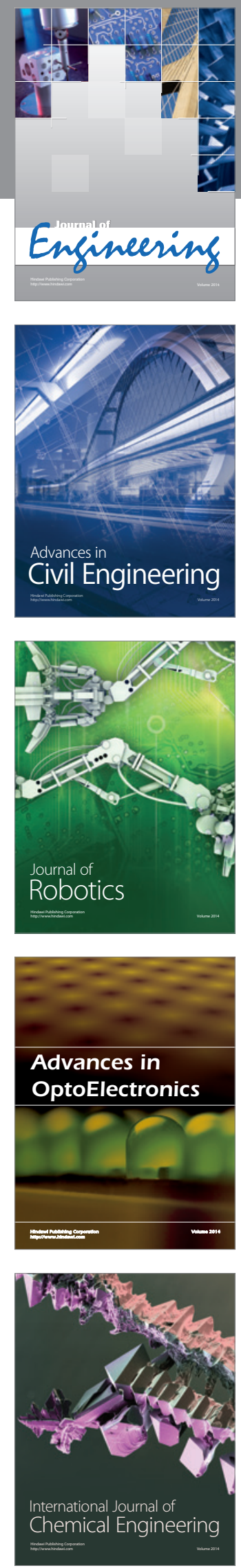

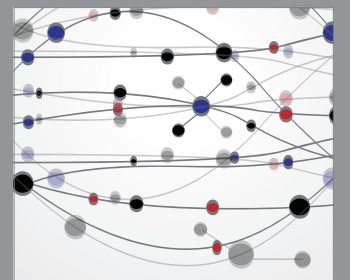

The Scientific World Journal
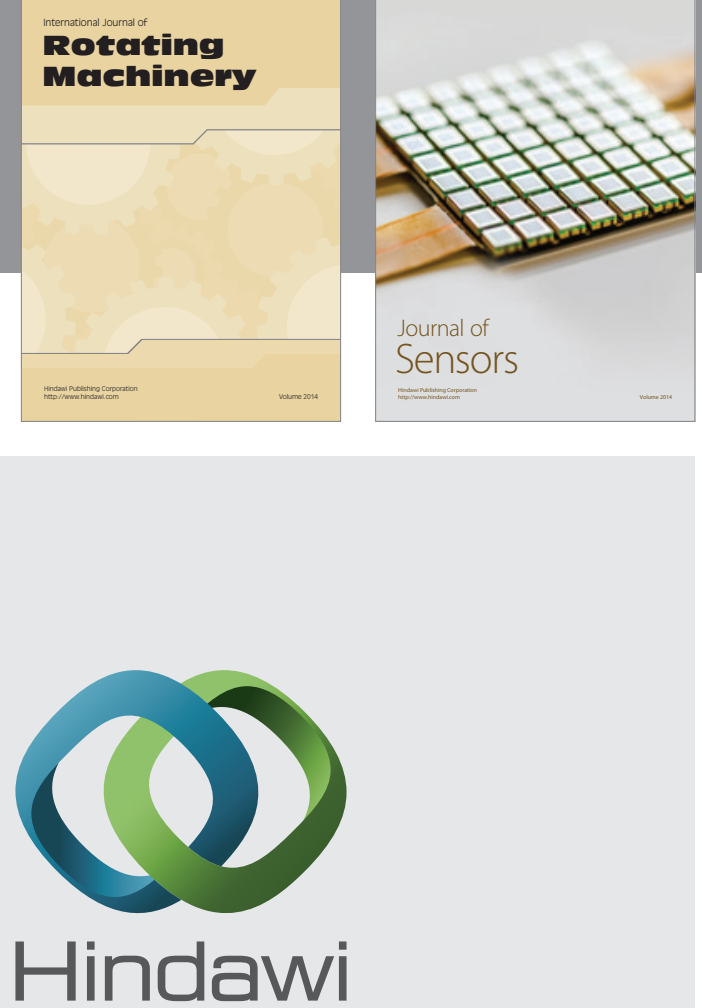

Submit your manuscripts at http://www.hindawi.com
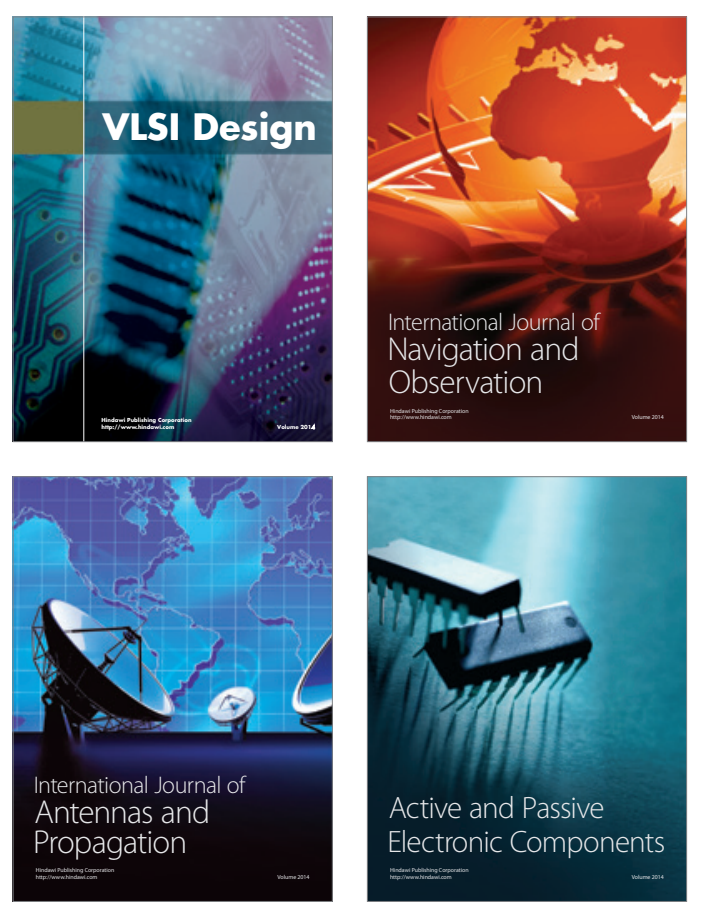
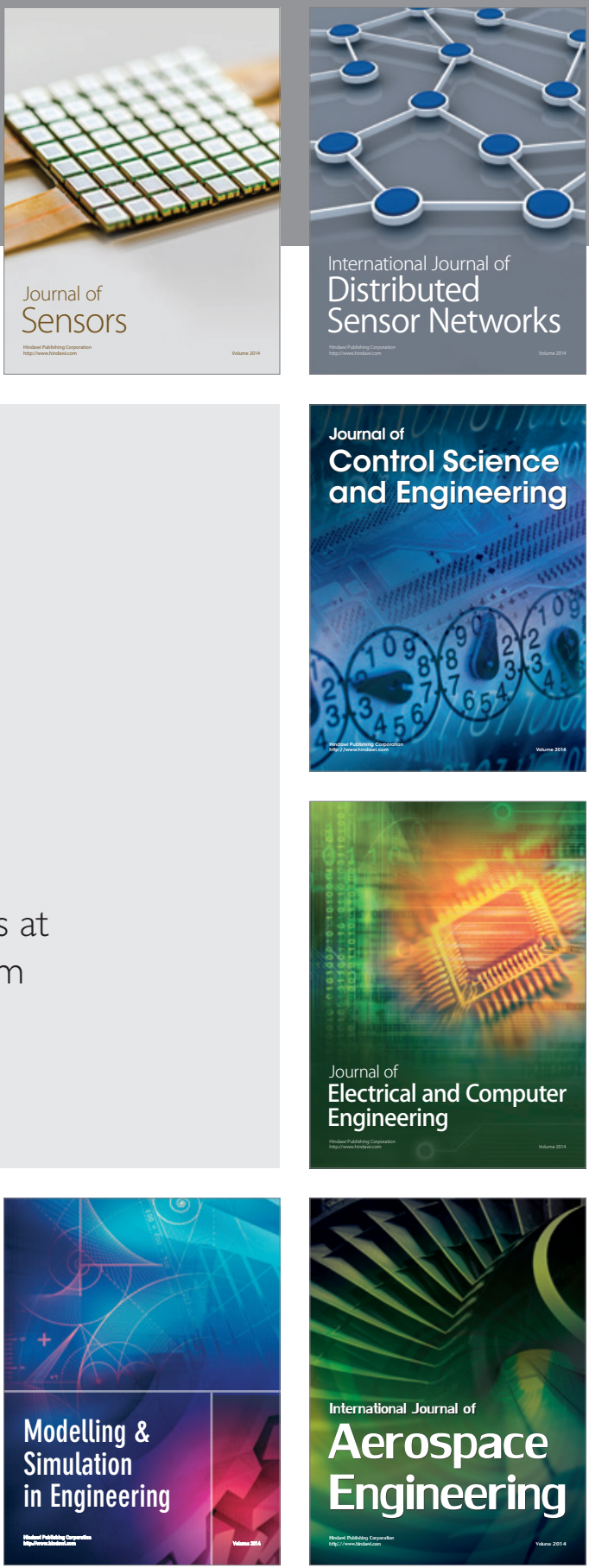

Journal of

Control Science

and Engineering
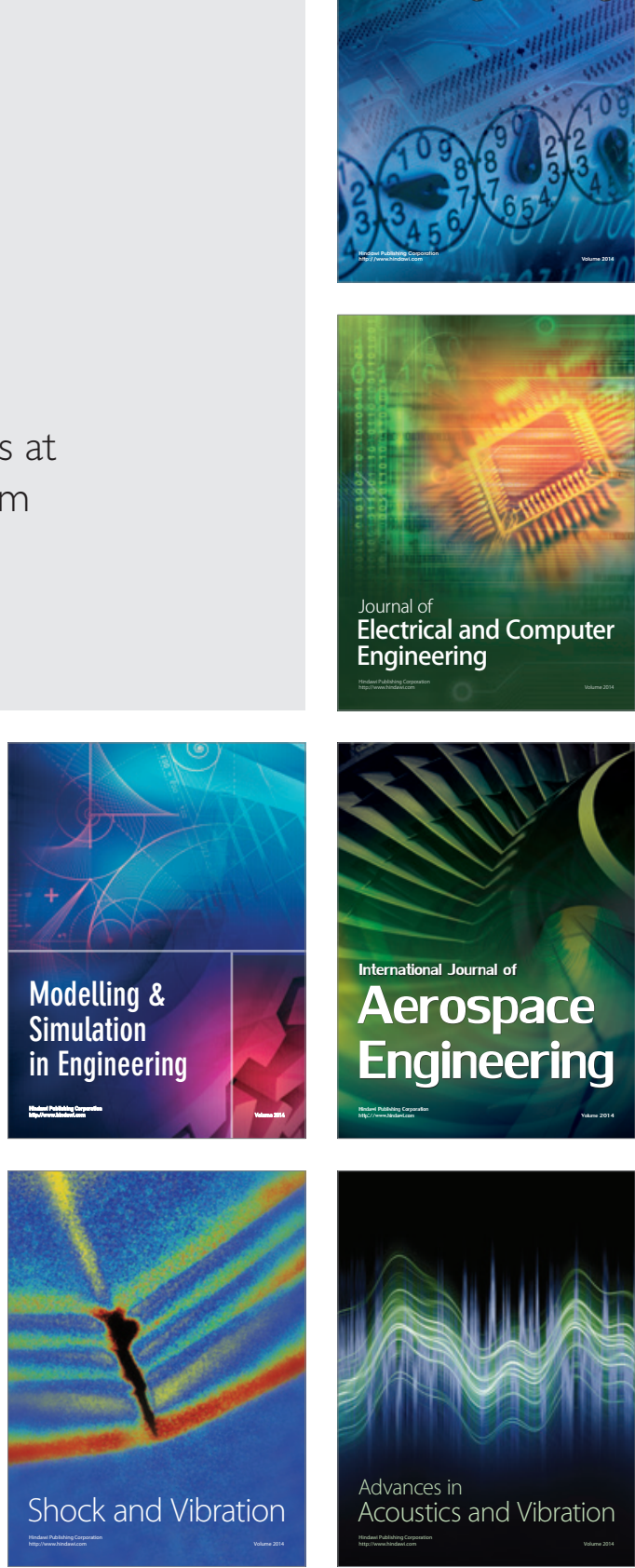